Dermatologische Zeitschrift. 1928;53:I-VIII

\title{
Contents, Vol. 53, 1928
}

\section{Inhaltsverzeichnis.}

Or $1 / 8$ inalarbeiten.

Albrecht, B. , Gclingt der Naehweis der Sypliilisinfektion bei Hund,

Schaf, Ziegc und Lama durch Drüseninfektion

Alexander, A., Zur Klizik seltener Soorformen 5

Armuzzi, G., Weitere ßeiträgc zur Frnge der Superiiifektion bei der experimentellcn Kaiiinchensyphilis $7 \Gamma>0$

Arzt, L· , Zur Frage der Antivirusbehandlung der Hauttuberkulose . . 12 Bergel, L. , Weitere Beiträge zur expcriincntellen Kaiiinchensypliilis . 21 Beron, B., Beitrag zur Behandlung der gonorrhoischen Gelenkeikiankungen mit monschliscliem Immunseruin 38 Bettmann, 8., Angiokeratome uncl keratotisclie Angiome 38

B1 u m e $\mathrm{nth}$ a 1 , F. und K. J a f fé, Versuche zur teclinischen Verbesserung der Serodiagnostik der Lues 54

Boas, H. und K. Novel, Vergleiehendc Untersuchungen über die $\Sigma$ und die Wassermann $\cdot$ Reaktion 63

Brill, E. , Zur Atiologie der Impetigo berpetiformis 70

Bruusgaard, E., Ein Fall von universeller Sklerodermie mit ausge-

breiteten Kalkablagerungen in dem kutanen Gewebe

- $\quad$ Ein Fall von Sarcomatosis cutis bei einem achtzehnjährigen Mädcben, von einem Ovarialsarkom ausgebend 86

Brünauer, St., Zur Histopathogenese der Comedonennaevi 91

Bruhns, C, Einige Bemerkungen über versehiedene Pilzarten und r'ilz-

nälirböden (Grütz-Agar, Pollacci-Agar) 104

Buschke, A. und W. Curtli, Über therapeutische Versuche mit Thal

lium, abgeselien von seiner Verwendung bei Pilzerkrankungen der

Kopfhaare 114

- $\quad$ und E. Langer, Zur Epidcmiologie und Behandlung der Pilz

erkrankungen 118

Cole, H. N., H. G. Miskjiau und J. Rauschkolb, Granuloma in-

guinale 127

Costa, S. Mendes da, Ein Fall von Akne nekrotica ausgedehnter Lo-

kalisation

144

Cronquist, C, Beitrag zur Belnuidluug der Pityriasis simplex capillitii

und des pityroden Haarausfalls

Dreyer, A., Über Salvarsanprophylaxe

149

Ehrmann, S., Über Sklerodermie 161

Frieboes, W., Granuloma multiplex soroti 164

Frühwald, E,., Ein Fall von Novokaindermatitis 
Fuhs, H., Zur Klinik der Freindkörpertumoren

183

- $\quad$ Über das seltene Syndrom von kongenitaleii Keratusen an Haut und

Kornea 199

Galewsky, E., Über Trichotillomanie 208

VI Inhaltsverzeíçhnis.

Soito

Gans, O., Endarteriitis oder Thromboangiitis obliterans 213

Grütz, 0., Sclcrodermia guttata follicularis mit halbseitîger Gesichts-

und Körperatrophie und endokrinen Kranklieitssymptoinen 227

(i ut in a n n, C, Wciteres über Amyloid der Haut 235

Habermann, R., Über Lichen ruber hyperthrophicub laevis und Lichen

obtusus 247

Hahn, F., Vom Quervy-Serum 546

Ilalberstaedter, L. und Alb. Simons, Über die Bchandlung der mit

Röntgenstrahlen nicht zú heilenden bösartigen Hautgeschwülst $\beta$. . 254 Пauck, L. , Beitrag zur

Ätiologie dor Folliculitis et Perifolliculitis

suffodiens et abscedens (E. Hoffmann) 275

Heller, J., Die Bccleutung der veränderten Wirtschaftsstruktur (Hoch-

und Spätkajiitalisnms) für die Statistik der Geschlechtskrankheiten 285 Iíerxlicimer, K. und Гr.

Herrmann, Über Fãrbu $\pi$, Vorkommen und

Punktion der eosinophilen Zellen 285

tleuck, W., Malariabehandlung bei Gonorrhoe $\quad 756$

Hoffmann, H., Über hautreizende Nutzhölzer 293

Hofmann, Edm., Über die Vererbung der Hydroa vacciniforme . . . 301

Jesionck, A., Haut und Tuberkelbazillen 310

Jordan, A., Sklero < lermie und Syphilis 327

Konrad, J, Unterstützung der Liehttherapic diirch die sensibilisierende

Wirkung des ïrypaflavins 331

Kilmer, L., Über die Beziehungen zwischen Herpes zoster generalisatus

und Varizellen 336

Leven, L., Erbbiologisches zur Lues 342

Linser, K, Zur externen Anwendung des Doramads (ThoriumX-Degea)

und seiner Dosíerung speziell in der Ekzemtherapie 348

Lipschütz, B., Über die Schwierigkeitcn der parasitologischen Pem-

phigusforschung $\quad 354$

Lomholt, Svend, Einige Worte über Syphilisbehandlung mit Salvarsan

und mit Wismut in Wassersuspension 368

Löhe, H. und H. Rosenfold, Über Monocytonangina mit anschließendem

vorübergehend seropositiven Erythema nodosum, zugleich ein Beitrag

zur Differenzialdiagnose zwischen luetischer und nichtlnetischer Angina 373

Lutz, W., Zur Kenntnis der Impetigo herpetiformis 383

Mayr, Julie K., Die Ergebnisse der Formolreaktion bei Dermatosen

in praktischer und theoretischer Bezichung 390

Memmesheimer, A. M., Zur Pathogenese der sogenannten essentiellon

Teleangiektasien 399 
Mergelsberg, 0., Über die Abortivkur der Lues I seronegativa . . 413 Moneorps, C, Über zwei Fälle wohlcharakterisierter Keratosen (Kera-

todermia punctata disseminata symmetrica, Cornu cutaneum) in ihrem Zusammenhaiig mit gleichzeitig bestehciider Lues 417

Müller, H., G. Sprenger und E. Uttendörfer, Bcratungsstelle, Fürsorge uncl Geschlechtskrankenabteilung der Stadt Mainz vor und nach Einführung des RGBG 427

Mulzer, P. und E. Keining, Übor die bei Sternborg-Paltaufscher

Krankheit vorkommenden speziíisclien und besondors unspezifischen Hautprozesse 438

Nathan, E. und Fr. Stern, Über den Mineralgehn.It der Haut ünter normalen und pathologischen Verhältnisseii. I. Mitteilung: Mikromethode zur quantitativen Bestimmung des Kalinin- und Kalziumgehaltes der Haut 451 ínhaltsverzeichnís. VII

S $\beta$ it $\beta$

Oelze, F. VV., Uber halbautomatiscbe ambulatorische Lumbalpunktion 457

Oppenheim, M., Über eine bei Gasarbeitern und Installateuren vor-

kommende typisclie Hautschädigung (Erfrierung, Einsprengung und

Exkoriationsbildung , 464

Polland, R., Über hämatogene, neurogene und psychogene Hautar $\prod \cdot k-$ tiouen 468

Ramel, E., Beitråge zur Kenntnis der Lymphogranulomatosis inguinalis 482 Reisner, A., Uber Krebsbildung auf dem ßoden eiues Lupus erythematosus 495

Ritter, H. und C. L. Karrenberg, Uber Hautschädigungen durch

Tragen von Pelzen 507

Rost, G. A. und Ph. Keller, Unserc Erfaliruogen mit diathermischer

Electrokoagulation in der Dermatologie 768

Rottmann, H. G., Zur Propbylaxe der kongenitalen Syphilis 516

Ruete, A. C., Lichen ruber planus pemphigoides $\quad 522$

Saalfeld, E., Ein ßeitrag zur Radiumnebonwirkung 533

Schmidt, W. J., Der submikroskopische Bau der Hornfibrillen, er-

schlossen aus der Polarisationsoptik des menschlichen Kopfhaares . 535

Schönfeld, W., Vaginale Resorptionsversuche 551

Schreus, H., Die Behandlung der Urticaria mit Alkali nebst Bemerkungen über den Säurebasenhaushalt bei Urtikaria und anderen Dermatosen 561

Scholtz, W., Über Wert und Spezifität der Tuberkulinreaktion bei Lupösen $\quad 775$

Schumacher, J., Salvarsanwirkung und Retikuloendothel 569

Schumacher, C. und R. Pachur, Experimentelle Untersuchungen und klinische Beobachtungen über Myosalvarsan778

Siemens, H. W., Zur klinisch-ätiologischen Analyse und Systematik kleinfteckiger Pïgme $\pi$ tierungen nebst Beschreibung einer besonderen Form von Epheliden 575 
Spiethoff, B., Blutbefunde bei Syphilis. Vor, unter und nach der Be handlung 592

Stein, R. 0., Herpes zoster generalisatus als Ursachc einer Varizellenepidemie 609

Strempel, R., Die Erhaltung der Pathogenität der Spir. pall, nach homologer Superinfektion des latent syphilitischen Kaninchens . . 615 Stümpke, Gr., Über Ulcus molle (Dmelcos) - Vakzine 620 Török, L., E. Rajka und E. Wessely, Stauung, Kapillardruck und Exsudation 628

Touton, K., Besonderheiten der Toxicodermia phylogenes 636 Unna, P. jun. und E. Delbanco, Zwei typisclie Fälle von Bowenscher Erkrankung 658

Valk, I. W. van der, Ein Beitrag zur ätiologischen Identilät der Im petigo bullosa. des Pemphigus neonatorum und der Dermatitis exfoliativa Ritter von Rittershain 668

Veiel, F., Perifolliculitis abscedens et surïodiens und ihre Beziehungen zur Acne conglobata 674

Volk, R., Periarteriitis nodosa bei einem Lupus erythematodes chron. cum exacerbatione $\quad 682$

Walthard, B., Zur Pathogenese des dysidrotischen Symptomenkomplexes. Über ein unter dem Bilde der Dysidrosis verlaufendes Epidermophytid 692

VIII Inhaltsverzeichnis.

Westphal, A. und Bach, Vorläufige Mitteiluug über die ersten hun-dert mit Malaria behandelten Fälle von progressiver Paralyse ... .

Wichmann, P., Die primäre exogene Infektion der Haut mit Tuberkulose in ihrer immunisatorischen Bedeutuug

$\Lambda$ Virz, Fıl, Zur Frage der „Lues nervosa”

Witteber, C, Über einen Fall syphilitischer Berufsinfektion am Unterschenkel (subkutane periphlebitische Initialsklerose)

Zurhelle, E. und 0. Schleyer, Weitere Studien über die Virulenz von syphilitischem Leichenvirus. (Vergleichende Impfungen mit frischem Virus bei demselben Versuchstier) 